\title{
Intraoperative Graft Isometry in Anatomic Single- Bundle Anterior Cruciate Ligament Reconstruction
}

\author{
You Keun Kim, $\mathrm{MD}^{1}$, Jae Doo Yoo, $\mathrm{MD}^{2}$, Sang Woo Kim, $\mathrm{MD}^{2}$, Sang Hoon Park, $\mathrm{MD}^{1}$, Joon Hee Cho, $\mathrm{MD}^{1}$, and \\ Hyung Mook Lim, $\mathrm{MD}^{3}$ \\ ${ }^{1}$ Department of Orthopaedic Surgery, National Police Hospital, Seoul; ${ }^{2}$ Department of Orthopaedic Surgery, Ewha Womans University Mokdong Hospital, Seoul; \\ ${ }^{3}$ Department of Orthopaedic Surgery, Shinchon Yonsei Hospital, Seoul, Korea
}

Purpose: Little is known about the isometry of anatomic single-bundle anterior cruciate ligament (ACL) tunnel positions in vivo although it is closely related to graft tension throughout the range of motion. The purpose of this study was to evaluate intraoperative graft isometry in anatomic singlebundle ACL reconstruction in vivo.

Materials and Methods: Graft length changes were assessed before bio-screw fixation in the tibial tunnel by pulling the graft with tensions of 20 lbs and $30 \mathrm{lbs}$ in full extension at flexion angles of $30^{\circ}, 60^{\circ}, 90^{\circ}$, and $120^{\circ}$.

Results: At the flexion angle of $30^{\circ}, 20 \mathrm{lbs}$ and $30 \mathrm{lbs}$ of tension showed $-0.4 \mathrm{~mm}$ and $-0.6 \mathrm{~mm}$ length changes, respectively. The greater the flexion angle of the knee, the shorter the graft length in the joint. At the flexion angles of $90^{\circ}$ and $120^{\circ}$, there was significant difference in the graft length change between $20 \mathrm{lbs}$ and $30 \mathrm{lbs}$ of tension.

Conclusions: Anatomic single-bundle ACL reconstruction was non-isometric. The graft length was the longest in full extension. The tension of graft became loose in flexion. At the flexion angles of $90^{\circ}$ and $120^{\circ}$, there was significant difference in the graft length change between $20 \mathrm{lbs}$ and $30 \mathrm{lbs}$ of tension.

Keywords: Knee, Anterior cruciate ligament, Reconstruction, Single bundle, Isometry

\section{Introduction}

The transtibial technique used to be the most popular method for creating the femoral tunnel in anterior cruciate ligament (ACL) reconstruction ${ }^{1}$. Although the femoral tunnel was originally placed at the so-called ACL isometric point with this technique, findings of detailed studies did not support the concept of ACL isometry ${ }^{2)}$. It is known to have significant drawbacks such as knee pain and rotatory instability due to separation from the

Received December 30, 2016; Revised (1st) April 13, 2017;

(2nd) August 6, 2017; (3rd) April 3, 2018; Accepted April 24, 2018

Correspondence to: Jae Doo Yoo, MD

Department of Orthopaedic Surgery, Ewha Womans University Mokdong

Hospital, 1071 Anyangcheon-ro, Yangcheon-gu, Seoul 07985, Korea

Tel: +82-2-2650-6142, Fax: +82-2-2650-0349

E-mail: koreanknee@gmail.com

This is an Open Access article distributed under the terms of the Creative Commons Attribution Non-Commercial License (http://creativecommons.org/licenses/by-nc/4.0/) which permits unrestricted non-commercial use, distribution, and reproduction in any medium, provided the original work is properly cited. real ACL femoral footprint caused by constraint in the direction of the tibial tunnel ${ }^{3)}$. As a result, changes in the surgical technique toward anatomic ACL reconstruction have been made over the past 10 years. Anatomic ACL reconstruction can be defined as functional restoration of the ACL to its native dimensions, collagen orientation, and insertion sites ${ }^{4)}$. In 2013, most (68\%) surgeons were using tibia-independent drilling techniques for making anatomic femoral tunnels in America ${ }^{5)}$. Many studies have reported that anatomic ACL reconstruction can restore knee function significantly and more closely to normal knee compared to non-anatomic procedures ${ }^{6,7)}$. However, little has been described about the isometry of anatomic single-bundle ACL tunnel positions in vivo, although it is closely related to graft tension throughout the range of motion $(\mathrm{ROM})^{8)}$. It is crucial for orthopedic surgeons to understand the effect of graft isometry in order to avoid graft failure which may occur when tensioning and fixation are done at an incorrect knee flexion angle. Therefore, the purpose of this study was to evaluate intraoperative graft isometry of anatomic single bundle ACL reconstruction with the 
outside-in technique and determine differences in isometry according to the tension during ACL graft fixation. Isometry was defined as less than $2 \mathrm{~mm}$ of length change during ROM. Our hypotheses of this study were as follows: (1) in anatomic singlebundle ACL reconstruction, length changes of graft during ROM would be isometric, and (2) there would be no difference in isometry between $20 \mathrm{lbs}$ and $30 \mathrm{lbs}$ of tension during ACL graft fixation.

\section{Materials and Methods}

\section{Subjects}

From October 2014 to June 2016, 60 patients with unilateral ACL deficiency who underwent anatomic single-bundle ACL reconstruction with autogenous hamstring graft were included in this study. ACL reconstruction was performed using the tibial tunnel-independent outside-in technique by a single surgeon (JDY). Consecutive patients for whom postoperative computed tomography (CT) scan was possible were enrolled in this study without randomization.

\section{Surgical Technique}

A routine arthroscopic examination of the knee joint was performed. After careful diagnostic arthroscopy, the tibial tunnel was prepared with the traditional transtibial technique. A femoral

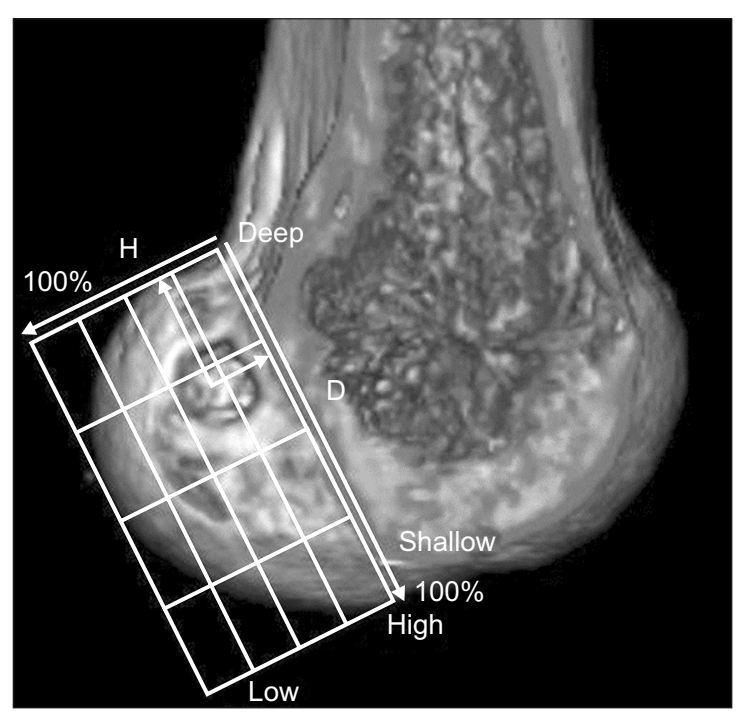

Fig. 1. Location of the center of the femoral tunnel defined as the percentage of the distance from the most posterior contour in reference to the total length of the lateral condyle parallel to the Blumensaat's line and the percentage of the distance from the intercondylar roof with respect to the total depth of the intercondylar notch perpendicular to the Blumensaat's line. H: perpendicular to the Blumensaat's line, D: parallel to the Blumensaat's line. socket was placed as close as possible to the center point of the ACL footprint referring to bony landmarks for direct insertion of the ACL graft. The femoral socket was made with the outsidein technique using retractable retrograde cutting bits (Flipcutter; Arthrex, Naples, FL, USA) which only required a portal-sized stab wound. After positioning the graft, TightRope (Arthrex) was used for femoral fixation of the graft. The graft on the tibial side was fixed at $10^{\circ}-20^{\circ}$ of flexion, using bioabsorbable interference screws. Additional fixation on the tibial side was performed using and a screw and a spiked washer").

\section{Three-Dimensional Reconstruction of CT}

Three days after the surgery, three-dimensional (3D) CT was performed with the knee extended. 3D CT images can depict bone tunnel apertures in three dimensions from all viewpoints ${ }^{10}$. 3D CT images were reconstructed to obtain sagittal, coronal, and axial plane views. For geometric measurement, the file was converted to DICOM format and transmitted to picture archiving and communication system (PiViewSTAR 5.0; INFINITT, Seoul, Korea).

\section{Tunnel Position on 3D CT}

The femoral tunnel position was assessed in postoperative $3 \mathrm{D}$ CT (Fig. 1). The quadrant method (Bernard quadrant method) was used to locate the femoral tunnel ${ }^{11}$. This method is commonly used with simple radiography and $3 \mathrm{D} \mathrm{CT} \mathrm{CT}^{12}$. On $3 \mathrm{D} \mathrm{CT}$ scans, a line connecting the most anterior and posterior edges

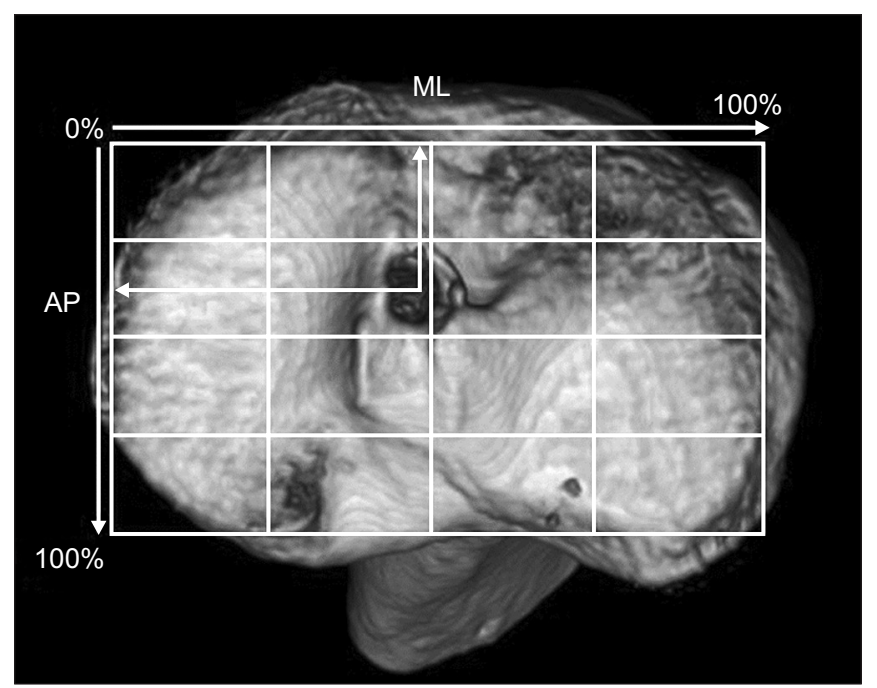

Fig. 2. Location of the center of the tibial tunnel defined as the percentage of the distance from the most medial contour with respect to the mediolateral (ML) width of the tibial plateau and the percentage of the distance from the most anterior contour with respect to the anteroposterior (AP) length of the tibial plateau. 
of the intercondylar roof was the reference for Blumensaat's line. The inferior border of the rectangle was a line tangent to the most distal point in the lateral condyle. Anterior and posterior edges of the lateral femoral condyle served as the other two borders to make a grid. ACL positioning was defined as a percentage of total sagittal diameter of lateral condyle and intercondylar notch height ${ }^{13)}$. To evaluate tibial tunnel position, a true proximal-todistal view on tibial plateau was used as described by Tsuda et al. ${ }^{14)}$ (Fig. 2). Femoral and tibial tunnel positions were assessed twice by two clinical knee fellows. Interobserver reliability was then determined.

\section{Intraoperative Graft Isometry Assessment}

The graft length change was assessed before bio-screw fixation in the tibial tunnel by pulling the graft with tensions of $20 \mathrm{lbs}$ and $30 \mathrm{lbs}$ in full extension at flexion angles of $30^{\circ}, 60^{\circ}, 90^{\circ}$, and $120^{\circ}$. The graft position was marked one centimeter apart from the outer orifice of tibial tunnel in full extension which was used as a reference length (Fig. 3). During flexion of the knee, the marked point of graft was identified. When the graft was moved outward, the graft length in the joint was shortened, resulting in a negative value of the graft length change. When the graft was moved inward, the graft length in the joint was lengthened, resulting in a positive value of the graft length change (Fig. 4). Graft length change was assessed twice by two clinical knee fellows. Interobserver reliability was then determined.

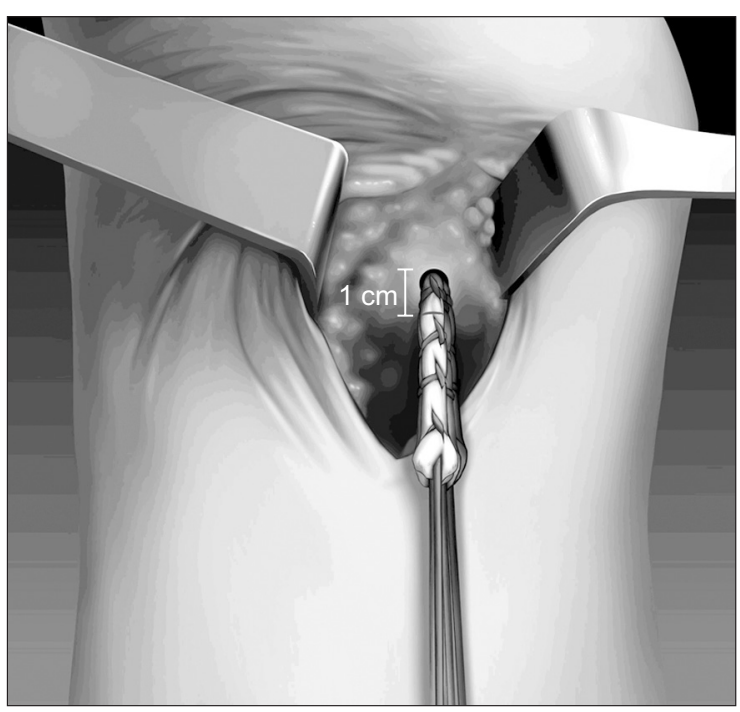

Fig. 3. Marking of graft position at one centimeter apart from the outer orifice of the tibial tunnel in full extension as a reference length.

\section{Statistical Analysis}

Interobserver reliability was determined using intraclass correlation coefficient (ICC) test. Interpretation of ICC was based on the criteria of Fleiss as follows: excellent, ICC $\geq 0.75$; fair-togood, $0.40 \leq \mathrm{ICC}<0.75$; and poor, ICC $<0.40$. Graft length changes at each flexion angle between tensions of $20 \mathrm{lbs}$ and $30 \mathrm{lbs}$ were compared by two-way repeated-measures of analysis of variance. All statistical analyses were performed using SPSS ver. 18.0 (SPSS Inc., Chicago, IL, USA).

\section{Results}

\section{Subjects}

A total of 60 patients were enrolled in the study, including 52 males and 8 females. Their mean age was 32.4 years (range, 15 to 56 years). Their mean height and body weight were $168 \pm 7 \mathrm{~cm}$ and $66 \pm 5 \mathrm{~kg}$, respectively.

\section{Tunnel Position}

The center of the femoral tunnel was located at $31.58 \pm 5.48 \%$ in the shallow/deep direction along Blumensaat's line and at $34.82 \pm 7.78 \%$ in the high/low direction from the intercondylar roof. The center of the tibial tunnel was located at $38.58 \pm 7.93 \%$ of the distance from the anterior edge of the tibia in anteroposterior direction and at $48.25 \pm 2.78 \%$ in mediolateral direction. This value is the average of measurements performed by the two

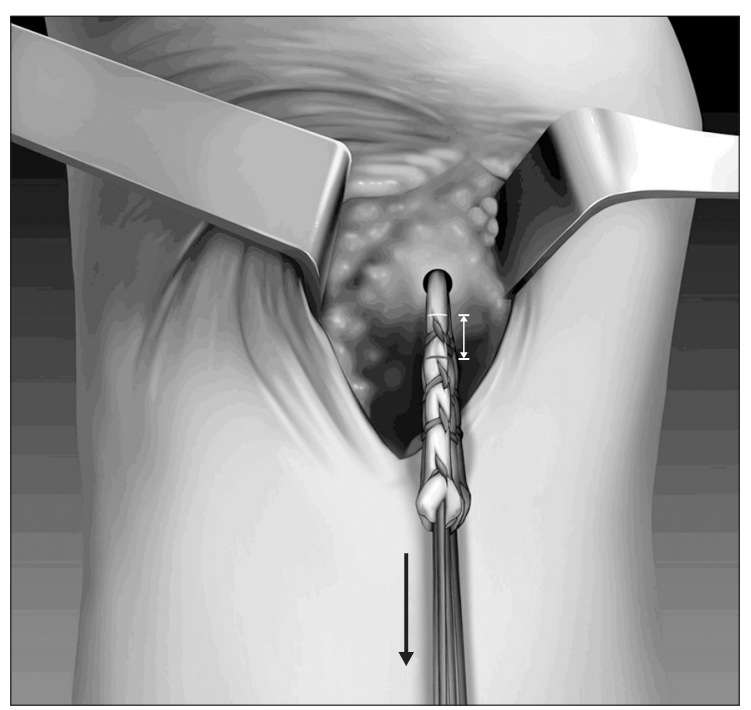

Fig. 4. Checking graft position change at each knee flexion angle. When the graft moves outward, the graft length in the joint is shortened, resulting in a negative value of graft length change. When the graft moves inward, the graft length in the joint is lengthened, resulting in a positive value of graft length change. 
Table 1. Length Change ( $\mathrm{mm}$ ) through Range of Motion with Tensions of $20 \mathrm{lbs}$ and $30 \mathrm{lbs}$

\begin{tabular}{lcccccc}
\hline \multirow{2}{*}{ Variable } & \multicolumn{5}{c}{ Flexion $\left(^{\circ}\right)$} & Maximum \\
\cline { 2 - 6 } & 0 & 30 & 60 & 90 & 120 & excursion \\
\hline $20 \mathrm{lb}$ & 0 & $-0.4 \pm 0.4$ & $-1.3 \pm 1.1$ & $-1.9 \pm 1.4$ & $-3.2 \pm 1.8$ & 3.2 \\
$30 \mathrm{lb}$ & 0 & $-0.6 \pm 0.5$ & $-1.5 \pm 0.9$ & $-2.4 \pm 1.5$ & $-3.5 \pm 1.6$ & 3.5 \\
p-value & 0.12 & 0.09 & $<0.05$ & $<0.05$ & $<0.05$ \\
\hline
\end{tabular}

Values are presented as mean \pm standard deviation.

clinical knee fellows. The ICC of interobserver reliability was 0.91 , indicating excellent reliability.

\section{Intraoperative Graft Isometry}

At $30^{\circ}$ of flexion angle, $20 \mathrm{lbs}$ and $30 \mathrm{lbs}$ of tension resulted in length changes of $-0.4 \mathrm{~mm}$ and $-0.6 \mathrm{~mm}$, respectively. The more the flexion angle of the knee, the shorter the graft length in the joint. At the flexion angles of $90^{\circ}$ and $120^{\circ}$, there was significant $(p<0.05)$ difference in the graft length change between $20 \mathrm{lbs}$ and $30 \mathrm{lbs}$ of tension. The ICC of interobserver reliability was 0.721 , indicating fair-to-good reliability (Table 1, Fig. 5).

\section{Discussion}

Graft isometry is relevant to graft tension during early postoperative phase, and it is also related to graft loosening. A cadaveric study has evaluated graft isometry in anatomic ACL reconstruction; however, a cadaveric knee model cannot reproduce muscle tension or tissue. Therefore, we evaluated isometry in anatomic ACL reconstruction in vivo. To the best of our knowledge, this is the first in vivo study that determines intraoperative graft isometry in anatomic ACL reconstruction. In this study, we defined a graft length change of less than $2 \mathrm{~mm}$ as isometric. According to this definition, the graft was isometric when the flexion angle of the knee was below $90^{\circ}$; it was non-isometric when the flexion angle was $90^{\circ}$ or over. However, some authors defined graft isometry as a length change of less than $1 \mathrm{~mm}$. According to such standard, the graft was isometric when flexion of the knee was less than $30^{\circ}$ while it was non-isometric when it was $30^{\circ}$ or above. Overall, anatomic single-bundle ACL reconstruction was non-isometric. The difference in graft length change between 20 lbs and $30 \mathrm{lbs}$ of tension was not statistically significant at knee flexion below $90^{\circ}$. However, there was significant difference in the graft length change between the two tension groups at knee flexion angles of $90^{\circ}$ or over. This indicates that the graft should be fixed using $20 \mathrm{lbs}$ of tension rather than $30 \mathrm{lbs}$ of tension to reduce the degree of non-isometry. In this study, 5 cases had a

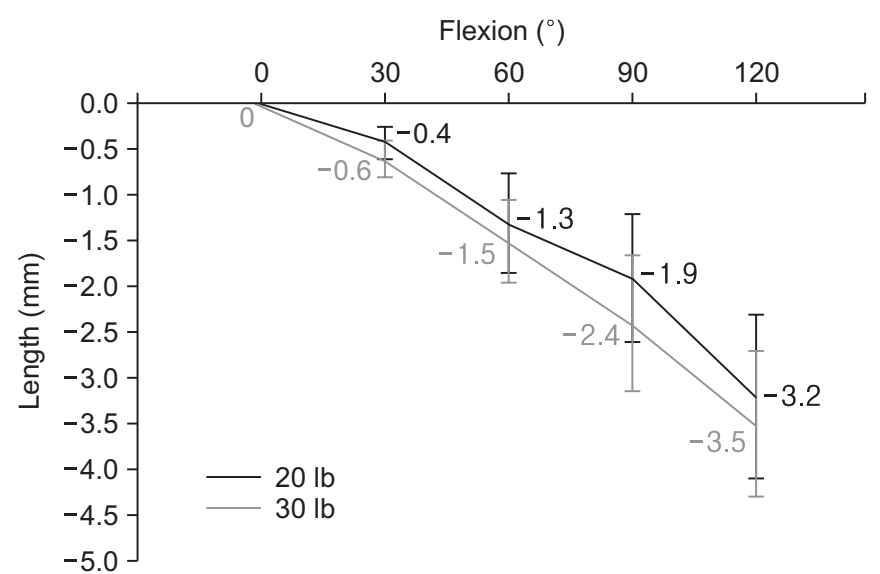

Fig. 5. Length change through range of motion with tensions of $20 \mathrm{lbs}$ and $30 \mathrm{lbs}$.

graft length change of more than $2 \mathrm{~mm}$ at the knee flexion angle of $30^{\circ}$. The mean graft length change was $2.3 \mathrm{~mm}$. The femoral tunnel was located at $39.2 \%$ in depth and $40.5 \%$ in height. This indicates that the femoral tunnel in the 5 cases was formed at shallower and lower positions than that in the other cases. A long-term follow-up is needed for these cases.

Anatomical placement of a femoral tunnel is very important in ACL reconstruction. Kim et al. ${ }^{15)}$ applied the Bernard quadrant method on 3D CT and found that it showed a high correlation with the conventional method using lateral radiographs. Therefore, the Bernard quadrant method can be used reliably for localizing a reconstructed ACL. This method was also used in this study to determine the femoral tunnel position. Colombet et al. ${ }^{16)}$ reported that the anteromedial bundle was located at $26.4 \%$ in the shallow/deep direction and $25.3 \%$ in the high/low direction while the posterolateral bundle was located at $32.3 \%$ of the length and $47.6 \%$ of the height in their cadaveric study. Zantop et al. ${ }^{17)}$ and Forsythe et al. ${ }^{18)}$ also reported similar results in their studies. In the present study, the femoral tunnel was located at $31.58 \%$ of the length and $34.82 \%$ of the height. These results were within the ranges of those reported in previous studies.

In the cadaveric study of Lee et al. ${ }^{8}$, the anatomic femoral tunnel was non-isometric and graft length was the longest in full extension. When a femoral anatomic tunnel was chosen for ACL reconstruction, the anterior tibial tunnel offered greater isometric benefits than the conventional tibial tunnel. Our results were in line with results of this cadaveric study. In another cadaveric study by Smith et al. ${ }^{19}$, , anatomic ACL positioning resulted in comparable anisometry to the native ACL. According to their results, a combination of a high anteromedial bundle femoral tunnel with an anteromedial bundle tibial tunnel resulted in the most 
isometric benefit among several options. Another important finding of this study was that ACL graft fixation in full extension would result in knee laxity. According to Lubowitz ${ }^{20)}$, ACL laxity during flexion can be better tolerated by patients than irreversible graft stretch and graft rupture or extension loss after ACL reconstructive surgery.

The strength of this study was that it was the first in vivo study that evaluated intraoperative graft isometry in anatomic singlebundle ACL reconstruction. However, this study has some limitations. First, there was a lack of data on correlation between intraoperative graft isometry and follow-up clinical study, which should be investigated in a further study. Second, there might be measurement errors due to small length changes.

\section{Conclusions}

Anatomic single-bundle ACL reconstruction is non-isometric: the graft length is the longest in full extension, and tension of graft becomes loose in flexion. At a knee flexion angle of $90^{\circ}$ or over, there was significant difference in graft length change between $20 \mathrm{lbs}$ of tension and $30 \mathrm{lbs}$ of tension. It would be appropriate to maintain a tension of $20 \mathrm{lbs}$ for graft fixation.

\section{Conflict of Interest}

No potential conflict of interest relevant to this article was reported.

\section{References}

1. Lee DH, Kim HJ, Ahn HS, Bin SI. Comparison of femur tunnel aperture location in patients undergoing transtibial and anatomical single-bundle anterior cruciate ligament reconstruction. Knee Surg Sports Traumatol Arthrosc. 2016; 24:3713-21.

2. Chung JY, Ha CW, Lee DH, Park YG, Park YB, Awe SI. Anatomic placement of the femoral tunnel by a modified transtibial technique using a large-offset femoral tunnel guide: a cadaveric study. Knee. 2016;23:659-65.

3. Lee MC, Seong SC, Lee S, Chang CB, Park YK, Jo H, Kim $\mathrm{CH}$. Vertical femoral tunnel placement results in rotational knee laxity after anterior cruciate ligament reconstruction. Arthroscopy. 2007;23:771-8.

4. van Eck CF, Lesniak BP, Schreiber VM, Fu FH. Anatomic single- and double-bundle anterior cruciate ligament reconstruction flowchart. Arthroscopy. 2010;26:258-68.
5. Chechik O, Amar E, Khashan M, Lador R, Eyal G, Gold A. An international survey on anterior cruciate ligament reconstruction practices. Int Orthop. 2013;37:201-6.

6. Yasuda K, van Eck CF, Hoshino Y, Fu FH, Tashman S. Anatomic single- and double-bundle anterior cruciate ligament reconstruction, part 1: basic science. Am J Sports Med. 2011; 39:1789-99.

7. Abebe ES, Utturkar GM, Taylor DC, Spritzer CE, Kim JP, Moorman CT 3rd, Garrett WE, DeFrate LE. The effects of femoral graft placement on in vivo knee kinematics after anterior cruciate ligament reconstruction. J Biomech. 2011;44: 924-9.

8. Lee JS, Kim TH, Kang SY, Lee SH, Jung YB, Koo S, Chang $\mathrm{SH}$, Lee WB, Jung HJ. How isometric are the anatomic femoral tunnel and the anterior tibial tunnel for anterior cruciate ligament reconstruction? Arthroscopy. 2012;28:1504-12.

9. Ko YW, Rhee SJ, Kim IW, Yoo JD. The correlation of tunnel position, orientation and tunnel enlargement in outsidein single-bundle anterior cruciate ligament reconstruction. Knee Surg Relat Res. 2015;27:247-54.

10. Ahn JH, Jeong HJ, Ko CS, Ko TS, Kim JH. Three-dimensional reconstruction computed tomography evaluation of tunnel location during single-bundle anterior cruciate ligament reconstruction: a comparison of transtibial and 2-incision tibial tunnel-independent techniques. Clin Orthop Surg. 2013;5:26-35.

11. Bernard M, Hertel P, Hornung H, Cierpinski T. Femoral insertion of the ACL: radiographic quadrant method. Am J Knee Surg. 1997;10:14-21.

12. Hoser C, Tecklenburg K, Kuenzel KH, Fink C. Postoperative evaluation of femoral tunnel position in ACL reconstruction: plain radiography versus computed tomography. Knee Surg Sports Traumatol Arthrosc. 2005;13:256-62.

13. Fernandes TL, Martins NM, Watai Fde A, Albuquerque C Jr, Pedrinelli A, Hernandez AJ. 3D computer tomography for measurement of femoral position in acl reconstruction. Acta Ortop Bras. 2015;23:11-5.

14. Tsuda E, Ishibashi Y, Fukuda A, Yamamoto Y, Tsukada H, Ono S. Tunnel position and relationship to postoperative knee laxity after double-bundle anterior cruciate ligament reconstruction with a transtibial technique. Am J Sports Med. 2010;38:698-706.

15. Kim DH, Lim WB, Cho SW, Lim CW, Jo S. Reliability of 3-dimensional computed tomography for application of the bernard quadrant method in femoral tunnel position evaluation after anatomic anterior cruciate ligament reconstruc- 
tion. Arthroscopy. 2016;32:1660-6.

16. Colombet P, Robinson J, Christel P, Franceschi JP, Djian P, Bellier G, Sbihi A. Morphology of anterior cruciate ligament attachments for anatomic reconstruction: a cadaveric dissection and radiographic study. Arthroscopy. 2006;22:984-92.

17. Zantop T, Wellmann M, Fu FH, Petersen W. Tunnel positioning of anteromedial and posterolateral bundles in anatomic anterior cruciate ligament reconstruction: anatomic and radiographic findings. Am J Sports Med. 2008;36:65-72.

18. Forsythe B, Kopf S, Wong AK, Martins CA, Anderst W, Tashman S, Fu FH. The location of femoral and tibial tun- nels in anatomic double-bundle anterior cruciate ligament reconstruction analyzed by three-dimensional computed tomography models. J Bone Joint Surg Am. 2010;92:1418-26.

19. Smith JO, Yasen S, Risebury MJ, Wilson AJ. Femoral and tibial tunnel positioning on graft isometry in anterior cruciate ligament reconstruction: a cadaveric study. J Orthop Surg (Hong Kong). 2014;22:318-24.

20. Lubowitz JH. Anatomic ACL reconstruction produces greater graft length change during knee range-of-motion than transtibial technique. Knee Surg Sports Traumatol Arthrosc. 2014;22:1190-5. 